\title{
Tratamiento de la investigación científica sobre los estudios de mujer, género y feminismo
}

\author{
María Eugenia Salvai \\ Universidad Nacional de Córdoba - UNC, Argentina
}

ARTÍCULO / ARTICLE

\begin{abstract}
Resumen
La investigación se propuso, luego de buscar en los catálogos de todas las bibliotecas de la Universidad Nacional de Córdoba, analizar el estado de la investigación elaborada desde la misma universidad, referida a estudios de mujer, género y feminismo entre los años 2000 y 2009. La hipótesis planteó que los documentos no resultan accesibles. En el presente artículo se analizan las posibles causas por las cuales la investigación sobre estudios de mujer, género y feminismo resulta invisibilizada.
\end{abstract}

Palabras clave

Estudios de mujer ; Estudios de género ; Feminismo ; Investigación científica ; Recuperación de la información ; Universidad Nacional de Córdoba

\section{Treatment of scientific research on women studies, gender and feminism}

\begin{abstract}
The purpose of the research was, after searching in the catalogues of all Universidad Nacional de Córdoba's libraries, analyze the state of gender, feminism and women studies' research elaborated from the university itself between years 2000 and 2009. The hypothesis planted that the documents are no access. this paper analyze the possible causes that make women studies, gender and feminism's research are invisibilized.
\end{abstract}

Keywords

Women studies ; Gender studies ; Feminism ; Scientific research ; Information retrieval ; Universidad Nacional de Córdoba

\section{Introducción}

En la actualidad la información es cada vez más valorada como fuente de poder, de allí que el manejo que se haga de ella resulta clave para el funcionamiento de cualquier organización. Comenzar a indagar sobre la literatura referida a los estudios de género a través de los distintos repositorios y directorios equivale a adentrarse en interminables laberintos, de los cuales no siempre se sale con un resultado positivo. ¿Qué lugar ocupan las investigaciones sobre mujer, género y feminismo en el universo de la producción científica elaborada en la Universidad Nacional de Córdoba (U.N.C.)? ¿Tiene la Universidad registro acerca de la producción científica referida a los estudios de mujer, género y feminismo? ¿En qué bibliotecas o centros de documentación se recoge y recupera esta información? ¿De qué manera se difunde la misma? La investigación planteada inicialmente como trabajo final de licenciatura en la carrera de Bibliotecología y Documentación, se propuso responder a estas preguntas. 
Isabel de Torres Ramírez (2005: p.480) afirma que: "En los umbrales del siglo XXI, pocas personas informadas dudarían de que los Estudios de las Mujeres (en adelante EEMM) constituyen una nueva área de conocimiento, que ha incorporado al saber y a la investigación nuevos objetos de estudio, perspectivas nuevas de análisis, nuevas preguntas e hipótesis de trabajo, contribuyendo, además, a crear un pensamiento crítico de gran proyección e influencia social". Por este motivo es que resultó útil conocer la producción científica referida a mujer, género y feminismo elaborada en la Universidad Nacional de Córdoba en la primera década del siglo XXI como así también su visibilidad, entendiendo como visibilidad la capacidad latente de los documentos de ser percibidos por un usuario determinado, o en este caso la capacidad de ser recuperados para su posterior consulta.

¿Por qué es importante conocer la producción referida a género y estudios de las mujeres? ¿Para qué sirve analizar la visibilidad de estos documentos? La respuesta podría ser: "porque sin información, no hay visibilidad, sin visibilidad, no hay prioridades" (Huyer, S. y Westholm, G., 2002). Dicho de otra forma: si no se conoce la realidad de la producción bibliográfica, no se pueden establecer prioridades ni políticas a partir de las cuales fortalecer el campo de estudio en análisis. Para poder documentar la presencia o la ausencia de los EMGF es necesario indagar la realidad, explicar la situación para a partir de allí proponer estrategias.

\section{Objetivos}

La investigación llevada a cabo se enmarcó dentro de los estudios bibliográficos, puesto que se buscó diagnosticar los obstáculos (si los hubiere) en la difusión de la investigación referida a mujer, género y feminismo. En relación a la delimitación semántica, en las estrategias de búsqueda se emplearon términos extraídos de dos tesauros: el Tesauro de la UNESCO ${ }^{1}$ y el Tesauro de género: lenguaje con equidad ${ }^{2}$. El análisis documental se restringió a la investigación de fuentes primarias de la información ya sean editadas o inéditas: artículos, libros, ponencias en congresos, publicaciones periódicas o seriadas, tesis (y todas sus variedades), trabajos de investigación, revisiones, etc. La delimitación espacial de este trabajo estuvo circunscripta al ámbito de la Universidad Nacional de Córdoba, abarcando todas las bibliotecas que dependen de la misma. La hipótesis planteó que "la escasa circulación y el desconocimiento hacen que la investigación referida a los estudios de mujer, género y feminismo se convierta en un producto documental de difícil recuperación y escasa accesibilidad". Históricamente se han invisibilizado los aportes a la sociedad y a la ciencia y la propia existencia de las mujeres como seres humanos, pero en este caso se podría hablar de una doble invisibilización: por un lado dicha invisibilidad histórica a la que han sido condenadas las mujeres; y por otro la provocada por la falta de mecanismos que permitan la creación y el mantenimiento de un acervo bibliográfico sobre la temática que asegure su accesibilidad en el tiempo.

El objetivo de la investigación fue analizar la situación en la que se encuentra la producción científica referida a los estudios de mujer, género y feminismo elaborada desde la Universidad Nacional de Córdoba (U.N.C.). Este propósito central implica los siguientes objetivos subsidiarios:

- Relevar la investigación científica elaborada en la Universidad Nacional de Córdoba en la primera década del siglo XXI referida a los estudios de mujer, género y feminismo.

- Identificar las distintas fuentes de producción de estudios de mujer y género en la Universidad Nacional de Córdoba.

\section{La Universidad Nacional de Córdoba}

La Universidad Nacional de Córdoba (UNC) es la más antigua de la Argentina, nace a principios del siglo XVII como Colegio Máximo el cual fundó la orden de los Jesuitas. En 1621 el Papa Gregorio XV le confiere la potestad de otorgar títulos de grado, derecho que fuera ratificado luego por Felipe IV en 1622 convirtiéndose en Universidad. La Compañía de Jesús estuvo a cargo de la casa de estudios hasta 1727, momento en que pasó a mano de los franciscanos. En el siglo XVIII se comienzan a dictar estudios de derecho. Según el Anuario Estadístico de 2009, la UNC está integrada por: facultades (12), centros de investigación (98), museos (16), colegios de nivel medio y 
terciario (2), centros de estudios de posgrado (3), bibliotecas (25), observatorios astronómicos (2), una editorial universitaria y un multimedio (radio AM, FM y dos canales televisivos).

\section{Los estudios de mujer, género y feminismo en la UNC}

Estudiar cualquier fenómeno con una mirada de género implica observar aquello que se encuentra invisibilizado, comenzar a cuestionar estructuras establecidas y naturalizadas a lo largo de siglos. Isabel de Torres Ramírez afirma que la perspectiva de género "permite una nueva visión de lo analizado, originando un saber también diferente, que reconoce "la sexuación del saber", es decir, evidencia que existen formas sexuadas de la cultura..." (2003: p.10). Gran parte de la información que se produce relacionada con los estudios de mujer y género se halla bajo la tipología de literatura gris. A esto respecto conviene citar a Isabel de Torres Ramírez (2000: p.4) quien afirma que es "una documentación dispersa, de difícil acceso, por tanto; multidisciplinar [...]; en aumento constante, se trata de un sector nuevo y emergente; difícil de recuperar y controlar, porque no existen todavía todos los instrumentos que sería necesario elaborar...". Son numerosas las ONG's que procesan periódicamente material tendiente a diseminar la visión de género entre la población. Diversos organismos gubernamentales elaboran reportes para prevenir la violencia de género o para informar acerca de la salud reproductiva. Muchas investigadoras viajan por el mundo presentando sus ponencias en congresos y conferencias. Asociaciones de mujeres producen guías tendientes a capacitar a otras mujeres en diversos aspectos. Esto solo para citar algunos ejemplos de la producción documental referida a estudios de mujer y género que se elabora en diversos ámbitos. Institucionalmente, en la UNC se dedican a la investigación de EMGF los siguientes grupos:

- Programa de Género dependiente de la Secretaría de Extensión Universitaria. Agrupa a docentes y personal investigador de la universidad vinculados a la temática de género.

- Programa Interdisciplinario de Estudios de Mujer y Género (PIEMG). Depende del Centro de Investigaciones María Saleme de Burnichón de la Facultad de Filosofía y Humanidades de la UNC.

- Programa de Género dependiente del Centro de Estudios Avanzados. Los antecedentes del programa pueden encontrarse en diversas actividades (de transferencia y de investigación) desarrollados en el marco del programa de Discurso Social, del Centro de Estudios Avanzados.

\section{Metodología}

En cuanto al diseño metodológico, se trató de un estudio de tipo exploratorio, descriptivo que tomó una década en sentido retrospectivo para lograr un análisis situacional en relación a la producción, circulación y recuperación del material referido a la temática. La metodología de trabajo consistió en analizar los catálogos de todas las bibliotecas dependientes de la Universidad Nacional de Córdoba. La búsqueda abarcó todos los centros, puesto que al considerarse el género como una categoría, atraviesa a todas las disciplinas. El objetivo fue analizar la situación en la que se encuentra la investigación científica referida a los estudios de mujer, género y feminismo elaborada desde la U.N.C. Se incluyeron no solo las bibliotecas de facultades, sino también las que dependen de Centros de Investigación o Institutos. Con el objetivo de unificar el vocabulario de búsqueda en los catálogos, se emplearon los términos propuestos por dos herramientas bibliotecológicas: el "Tesauro UNESCO" y el "Tesauro de género: lenguaje con equidad", elaborado por el Instituto Nacional de las Mujeres de México. En ambos se buscaron los siguientes términos genéricos y sus respectivos términos específicos: Género, Estudios de género, Teoría de género, Perspectiva de género, Feminismo, Estudios de las mujeres, Discriminación de género/Discriminación sexual y Violencia de género/violencia conyugal (Cuadro 1). 


\begin{tabular}{lll}
\hline Término buscado & Tesauro UNESCO & Tesauro de género \\
\hline GÉNERO & $\begin{array}{l}\text { Género humano (Use Especie } \\
\text { humana*) } \\
\text { *Se eliminó de la búsqueda } \\
\text { posterior por su amplitud }\end{array}$ & $\begin{array}{l}\text { Género* } \\
{ }^{*} \text { Se eliminó de la búsqueda } \\
\text { posterior por su amplitud }\end{array}$ \\
\hline ESTUDIOS DE GÉNERO & Sin repuesta & Estudios de género \\
\hline TEORIA DE GÉNERO & Sin respuesta & Teorías de género \\
\hline PERSPECTIVA DE & Sin respuesta & Perspectiva de género \\
GÉNERO & $\begin{array}{l}\text { Feminismo (Use Movimiento } \\
\text { de liberación femenina) }\end{array}$ & Feminismo \\
\hline FEMINISMO & Sin respuesta & Estudios de la mujer \\
\hline ESTUDIOS DE LA MUJER & Mujer & Sin respuesta \\
\hline MUJER & Sin respuesta & Mujeres \\
\hline MUJERES & Sin respuesta & Discriminación de género \\
\hline DISCRIMINACIÓN DE \\
GÉNERO & Discriminación sexual & Discriminación sexual \\
\hline DISCRIMINACIÓN & Sin respuesta & Violencia de género \\
SEXUAL & $\begin{array}{l}\text { Violencia conyugal } \\
\text { (Use Violencia doméstica) }\end{array}$ & Sin respuesta \\
\hline VIOLENCIA DE GÉNERO & $\begin{array}{l}\text { Violencia familiar } \\
\text { (Use Violencia doméstica) }\end{array}$ & Sin respuesta \\
\hline VIOLENCIA FAMILIAR & CONYUGAL &
\end{tabular}

Luego de la unificación de los resultados obtenidos de ambos tesauros, se obtuvo el siguiente listado de términos: Estudios de género; Estudios de la mujer; Teorías de género; Perspectiva de género; Feminismo; Teoría Feminista; Movimiento de liberación femenina; Organización femenina; Mujer/Mujeres; Discriminación de género; Discriminación sexual; Discriminación contra la mujer; Derechos de la mujer; Violencia de género; Violencia contra las mujeres; Violencia doméstica. En un primer momento de la búsqueda se realizó a través de los catálogos en línea de las bibliotecas; siempre que las interfaces de consulta lo permitieran, se seleccionó la opción de búsqueda por palabras clave. Cuando no pudo realizarse la búsqueda en línea, se consultó personalmente el catálogo de cada institución.

\section{Resultados}

Cabe recordar que para que un registro fuera considerado a los fines de esta investigación debía cumplir con una serie de requisitos:

1. Que alguno de los descriptores contenidos en el registro coincidiera con los propuestos por esta investigación,

2. Que fuera un documento elaborado desde la UNC, es decir que en el campo autor, autor institucional o editor apareciera la Universidad.

3. Y que estuviera comprendido en el periodo de tiempo limitado entre 2000 y 2009.

Se muestran a continuación los resultados obtenidos de las búsquedas (Cuadro 2). 


\begin{tabular}{ll}
\hline \multicolumn{1}{c}{ Biblioteca } & Coincidencias totales $^{*}$ \\
\hline Biblioteca del Observatorio Astronómico & 0 \\
\hline Biblioteca Hemoderivados & 0 \\
\hline $\begin{array}{l}\text { Biblioteca "Mario Fernández Ordóñez" - Facultad de } \\
\text { Arquitectura, Urbanismo y Diseño }\end{array}$ & 0 \\
\hline Biblioteca Escuela de Graduados FAUD & 0 \\
\hline $\begin{array}{l}\text { Biblioteca "Ing. Agr. Moisés Farber" - Facultad de } \\
\text { Ciencias Agropecuarias }\end{array}$ & 0 \\
\hline $\begin{array}{l}\text { Biblioteca "Manuel Belgrano" - Facultad de Ciencias } \\
\text { Económicas }\end{array}$ & 0 \\
\hline $\begin{array}{l}\text { Biblioteca Ing. Ríos e Ing. Achával - Facultad de } \\
\text { Ciencias Exactas, Físicas y Naturales }\end{array}$ & 0 \\
\hline $\begin{array}{l}\text { Biblioteca "Dr. Aníbal A. Sanguinetti" - Facultad de } \\
\text { Ciencias Químicas }\end{array}$ & 0 \\
\hline Biblioteca - Escuela de Ciencias de la Información & 0 \\
\hline Biblioteca - Escuela de Artes & 0 \\
\hline $\begin{array}{l}\text { Biblioteca "Daniel Sonsini" - Facultad de Matemática, } \\
\text { Astronomía y Física }\end{array}$ & 0 \\
\hline $\begin{array}{l}\text { Biblioteca Escuela Superior de Comercio Manuel } \\
\text { Belgrano }\end{array}$ & 0 \\
\hline Biblioteca del Colegio Nacional de Montserrat & 1 \\
\hline Biblioteca Dr. Lutti - Facultad de Ciencias Exactas, \\
\begin{tabular}{l} 
Físicas y Naturales \\
\hline Biblioteca "José M. Aricó"
\end{tabular} \\
\hline Biblioteca - Facultad de Odontología & 1 \\
\hline Biblioteca - Escuela de Nutrición & 10 \\
\hline Biblioteca "María A. Suárez de Hünicken" -Escuela de \\
Enfermería
\end{tabular}

Del total de catálogos de bibliotecas analizados, no se obtuvo ningún resultado en 13, lo que implica que en el 50\% de los catálogos no se encontró información relacionada con los EMGF elaborada por la UNC entre 2000 y 2009. Del $50 \%$ que se obtuvieron resultados positivos, ¿cuál es la distribución? (Figura №1):

- Catálogos con entre 1 a 5 coincidencias: Biblioteca de la Facultad de Ciencias Exactas, Físicas y Naturales (sede centro), Biblioteca Escuela de Enfermería, Biblioteca de la Escuela de Salud Pública, Biblioteca de la Escuela de Nutrición, Biblioteca Aricó, Biblioteca de la Escuela de Trabajo Social y Biblioteca de la Facultad de Odontología. 
- Catálogos con entre 6 a 10 coincidencias: Biblioteca Mayor, Biblioteca del CEA, Biblioteca de la Facultad de Derecho y Ciencias Sociales y Biblioteca de la Facultad de Ciencias Médicas.

- Catálogos con entre 11 a 15 coincidencias: Biblioteca de la Facultad de Lenguas.

- Catálogos con más de 16 coincidencias: Biblioteca de las Facultades de Filosofía y Humanidades y Psicología.

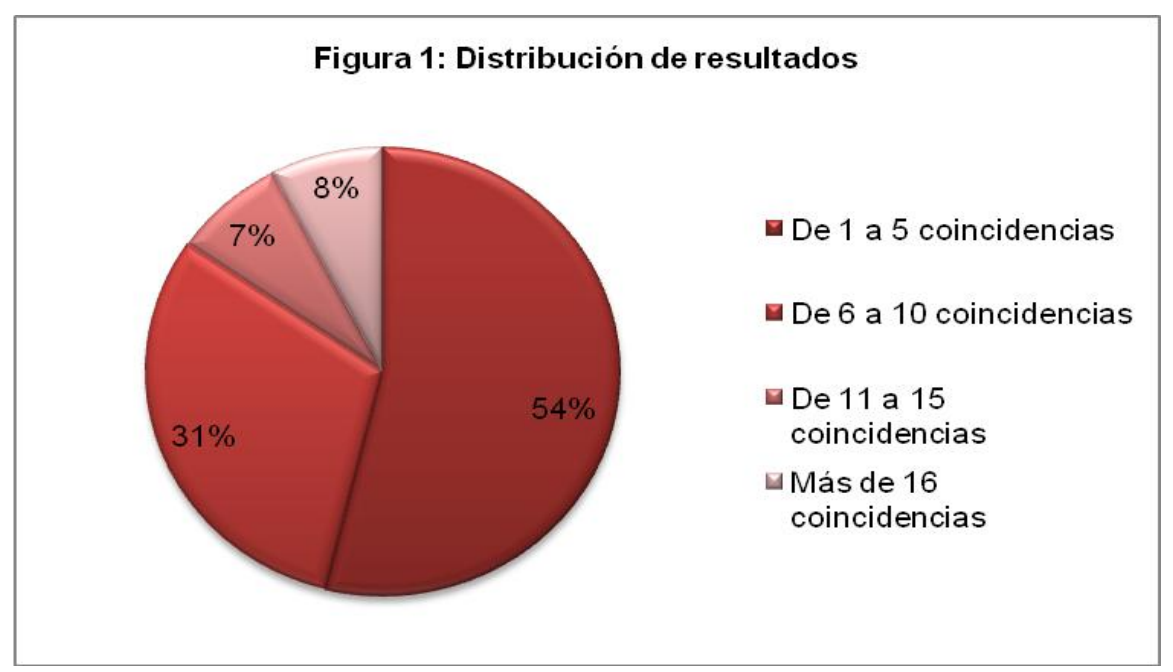

Puede notarse que gran parte de los resultados positivos (entendiéndose como resultado positivo cada hallazgo que coincidiera con las expresiones de búsqueda) fueron encontrados en bibliotecas que poseen un perfil más afín con las ciencias sociales o humanas, en tanto que en las llamadas bibliotecas pertenecientes a las ciencias duras, las coincidencias fueron escasas.

A partir de estos datos puede analizarse cuáles son las facultades (y los respectivos campos del saber) en los cuales los estudios de mujer, género y feminismo poseen mayor presencia. Para esta organización de la información se empleará la clasificación en grandes áreas del saber utilizada por la universidad en la presentación de sus anuarios estadísticos. Estas áreas son: ciencias básicas y aplicadas (abarca las facultades de Arquitectura, Urbanismo y Diseño, Facultad de Ciencias Exactas, Físicas y Naturales; Facultad de Matemática, Astronomía y Física; Facultad de Ciencias Químicas y Facultad de Ciencias Agronómicas), ciencias sociales (abarca Facultad de Derecho y Ciencias sociales; Facultad de Ciencias Económicas; se incluyen por temática las bibliotecas José M. Aricó, Biblioteca Mayor y Biblioteca y Centro de Documentación Latinoamericano de CEA y las bibliotecas de las escuelas medias dependientes de la universidad), ciencias médicas (agrupa a Facultad de Ciencias Médicas con sus respectivas bibliotecas de escuelas y Facultad de Odontología, se incluye aquí a la biblioteca de Hemoderivados) y ciencias humanas (con la Facultad de Filosofía y Humanidades, Facultad de Psicología y Facultad de Lenguas). 


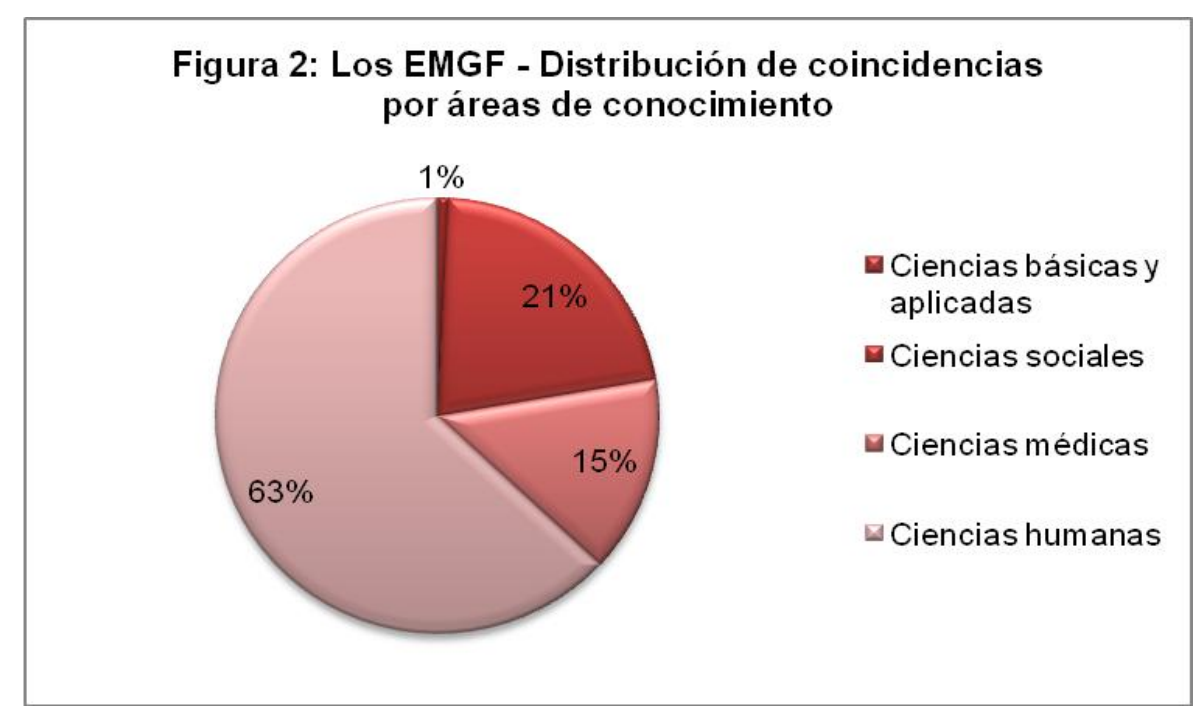

Puede observarse claramente (Figura №2) que las áreas más productivas respecto a los EMGF en lo referente a la producción de material bibliográfico son en primer lugar el área de Humanidades (con un $63 \%$ del total de la producción) seguida luego por las ciencias sociales (con un 21\%). A partir del mismo gráfico y a pesar de las diferencias notorias que existen entre las áreas, puede verse que los estudios de mujer, género y feminismo de alguna manera están presentes en todos los campos del saber.

De una segunda observación puede concluirse que se han encontrado gran variedad de fuentes de información: libros, actas de congresos, tesis de grado, de posgrado, publicaciones periódicas, entre otras (Cuadro №3). Esto viene a reforzar la idea de que los EMGF son no solo multidisciplinarias como pudo observarse a partir de la variedad de bibliotecas en las que se halló material, sino también variados en la elección de los soportes que contienen la información.

Cuadro 3: Tipología documental

\begin{tabular}{lr}
\hline Tipo de publicación & Cantidad \\
\hline Libros & 29 \\
\hline Congresos/Actas & 16 \\
\hline Tesis de posgrado & 19 \\
\hline Artículos de revista & 1 \\
\hline Tesis doctorales & 2 \\
\hline Tesinas & 1 \\
\hline Tesis de grado & 2 \\
\hline Trabajos Finales de Licenciatura & 57 \\
\hline Cd-rom & 1 \\
\hline Publicaciones periodicas & 2 \\
\hline Total & $\mathbf{1 3 0}$ \\
\hline
\end{tabular}


Figura 3: Porcentajes de fuentes de información encontradas

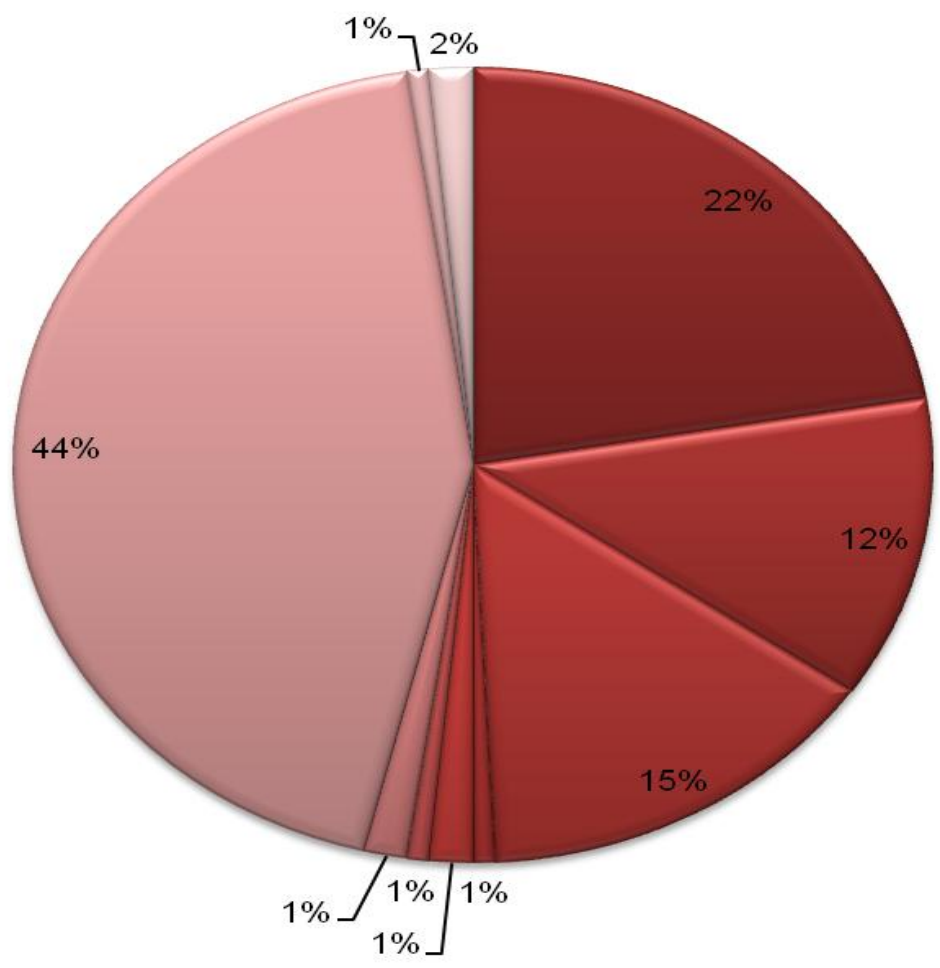

Libros

- Congresos/Actas

- Tesis de posgrado

Artículos de revista

- Tesis doctorales

Tesinas

$\square$ Tesis de grado

$\square$ Trabajos Finales de Licenciatura

$\triangle \mathrm{Cd}$-rom

$\triangle$ Publicaciones periodicas

Al momento de discriminar la información encontrada, se respetó la designación dada a cada trabajo de investigación exigido por cada facultad. En algunos casos se trata de trabajos finales de licenciatura, en otro se los considera tesinas o tesis de grado. Más allá de la designación empleada, se trata de trabajos de investigación necesarios para obtener un título de grado. Este hallazgo confirma la afirmación de Isabel de Torres Ramírez acerca de la dificultad en la identificación y localización de gran parte de la documentación sobre EMGF, ya que este tipo de escritos carecen de control bibliográfico y no circulan de manera comercial.

Si se agrupan bajo el concepto de escritos académicos todos aquellos trabajos de investigación exigidos por las facultades para la obtención de un título de grado o posgrado, se está ante 81 trabajos, es decir el 62\% de la producción (se obtiene de sumar los trabajos finales de licenciatura, las tesis de posgrado, y las tesis doctorales, tesinas y tesis de grado). Ahora bien, deteniéndonos en lo que implica haber encontrado un porcentaje tan alto de escritos académicos, podríamos decir que resulta muy positivo por varios motivos: en primer lugar puede observarse que existen estudiantes interesados/as en la temática (EMGF) que eligen desarrollar sus trabajos de investigación sobre esta disciplina. En segundo lugar, se evidencia la presencia de un campo de futuros profesionales (y potenciales docentes o investigadores/as) de la universidad con un manifiesto interés por los EMGF, espacio que puede ser aprovechado por la casa de estudios para desarrollar aun más sus trabajos en esta área de investigación.

Al ordenar los resultados en base a las cantidades (Figura №3) puede verse que la mayoría pertenecen a la tipología de la literatura gris. Este hallazgo confirma la afirmación de Isabel de Torres Ramírez acerca de la dificultad en la identificación y localización de gran parte de la documentación sobre EMGF, ya que este tipo de escritos carecen de control bibliográfico y no circulan de manera comercial. 


\begin{tabular}{|c|c|c|c|c|}
\hline Tipo de literatura & Forma documental & Cantidad & Total & \\
\hline \multirow[t]{7}{*}{ Literatura gris } & & & 97 & $74,61 \%$ \\
\hline & Congresos/Actas & 16 & & \\
\hline & Tesis de posgrado & 19 & & \\
\hline & Tesis doctorales & 2 & & \\
\hline & Tesinas & 1 & & \\
\hline & Tesis de grado & 2 & & \\
\hline & Trabajos Finales de Licenciatura & 57 & & \\
\hline \multirow[t]{6}{*}{ Literatura convencional } & & & 33 & $25,39 \%$ \\
\hline & Libros & 29 & & \\
\hline & Artículos de revista & 1 & & \\
\hline & Cd-rom & 1 & & \\
\hline & Publicaciones periódicas & 2 & & \\
\hline & & Total & 130 & $100,00 \%$ \\
\hline
\end{tabular}

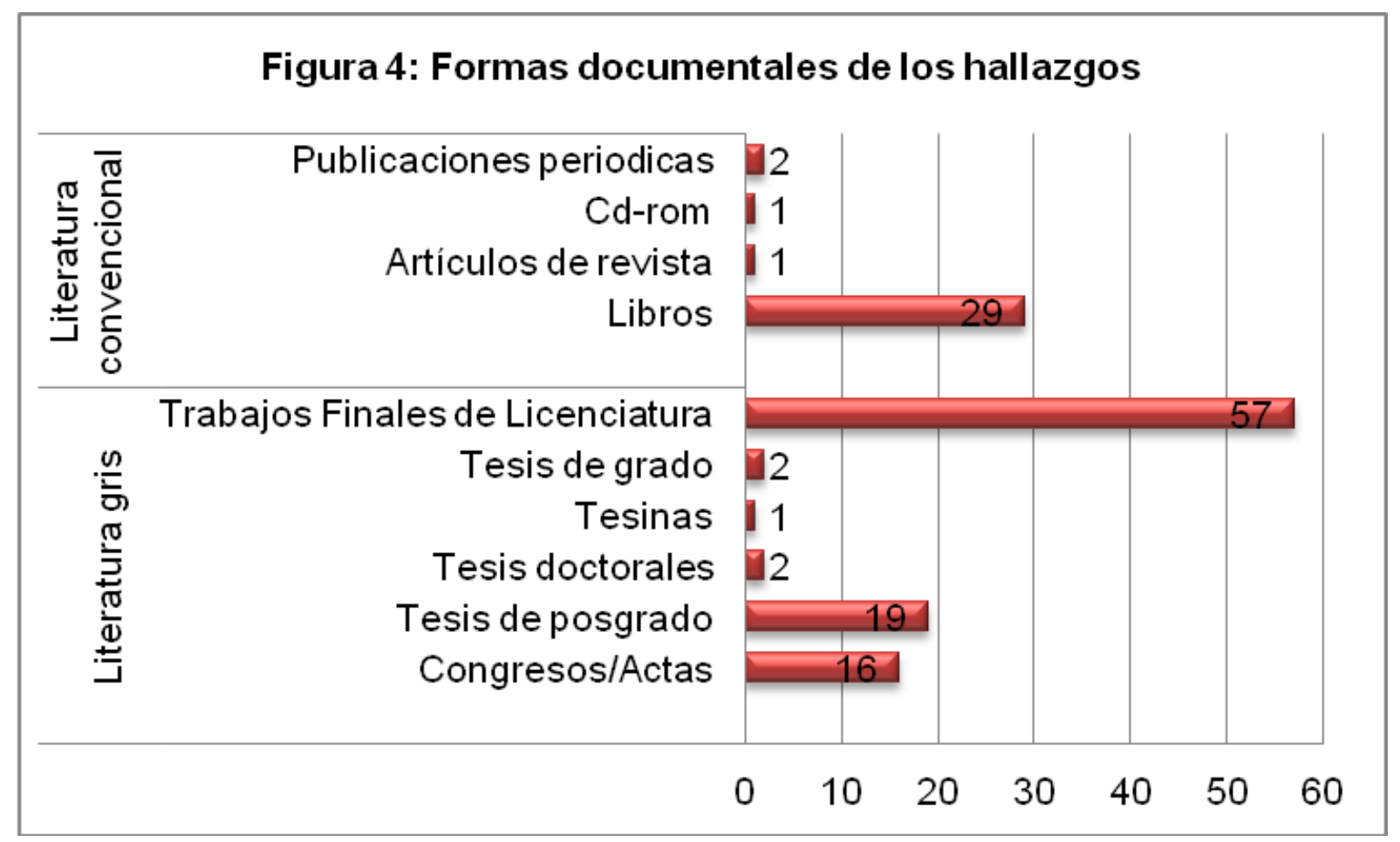




\section{Conclusiones}

No caben dudas de que en la UNC se investiga acerca de estudios de mujer y género, el tema está presente y cobrando cada día mas fuerza. Podría decirse que la temática está en crecimiento. Con los términos empleados en la búsqueda ¿se han recuperado todos los documentos elaborados desde la UNC entre 2000 y 2009 sobre la temática de estudios de género, mujer y feminismo? La respuesta es seguramente negativa. Suponiendo que no sea posible recuperar toda la información ¿se han podido identificar en los catálogos un buen número de registros? Esta pregunta es difícil de responder ya que se está ante una realidad que en cierta manera obstaculiza la visibilidad de la información. ¿A qué se hace referencia con esta realidad obstaculizadora? A partir de la información recopilada se pueden identificar varios escollos en el camino entre el documento y su autor/a y la biblioteca. Estas pérdidas obedecen a diferentes motivos.

Al inicio de la investigación se planteó la siguiente hipótesis: la escasa circulación y el desconocimiento hacen que la investigación referida a los estudios de mujer, género y feminismo se convierta en un producto documental de difícil recuperación y escasa accesibilidad. De las cifras recolectadas, se evidencia esta situación. Es decir: los documentos no circulan porque no llegan a las organizaciones destinadas para ese fin, las bibliotecas. Si se implementaran una serie de políticas que aseguraran la conservación firme de al menos un ejemplar de cada documento, y si esos documentos recibieran un correcto tratamiento los/as interesados/as podrían acceder de manera sencilla a la información, y no solo se garantizaría el acceso si no también la visibilidad ante otras unidades educativas, por ejemplo. El planteo de la hipótesis hablaba de la escasa circulación y el desconocimiento. Este segundo aspecto implica la labor bibliotecaria en sí. La temática de EMGF es específica, multidisciplinaria y emergente, por este motivo es necesario que sean objeto de un análisis y una descripción exhaustiva, con los términos adecuados para asegurar su identificación y recuperación por parte de los/as interesados/as. El feminismo se ha preocupado por demostrar que los conocimientos tradicionalmente aceptados responden a una concepción patriarcal del universo, para contrarrestar esta impronta es necesario generar nuevos conocimientos. Esta es otra gran característica del movimiento feminista: cuestionar algo y reemplazarlo, deconstruir para construir una nueva concepción. ¿Están preparadas las unidades de información para recibir y tratar estos documentos? Para garantizar la divulgación y posterior creación de nuevos conocimientos, es necesario asegurar en primer lugar la identificación, localización y posterior accesibilidad de ese material. Ante temáticas tan específicas como lo son los EMGF es importante para el análisis documental, contar con herramientas idóneas, por ejemplo utilizar tesauros o listas de encabezamiento de materia especializados en el tema, de esta manera, la biblioteca se asegura un correcto tratamiento de los documentos. Otra opción enriquecedora puede ser recurrir a los/as investigadores/as expertos/as en la disciplina.

Las herramientas con las que se asignan descriptores a los documentos referidos a los estudios de mujer, género y feminismo no resultan del todo apropiadas. El Tesauro de UNESCO, como se ha demostrado en esta investigación ofrece pocos términos para describir el material, y los que aparecen resultan demasiado genéricos. En una disciplina tan específica como lo son los EMGF, en constante crecimiento además, cuyo corpus teórico está en discusión y elaboración, es necesario utilizar herramientas que estén a la altura de las demandas planteadas por los documentos.

El bibliotecario Sandford Berman ${ }^{3}$ ha propuesto una serie de condiciones que debe cumplir el catálogo de una biblioteca pública, si bien en esta investigación se ha hablado de bibliotecas universitarias, el concepto puede trasladarse de manera acertada. Los principios de Berman son:

- Inteligibilidad: es decir tanto el formato del catálogo como el contenido deben poder ser entendidos por los/as usuarios/as.

- Recuperabilidad: el lenguaje usado para describir los documentos debe ser contemporáneo y familiar para quienes consultan. Se deben incluir herramientas que ayuden la consulta como lo son las referencias de "véase" y "véase además". 
- Equidad: se debe respetar el contenido del documento. Los descriptores empleados para representar conceptos de edad, género, raza, religión, etc. deben respetar las designaciones propias del grupo.

Cumplir con estas condiciones colaboraría de gran manera, con la visibilidad y la recuperabilidad de la documentación referida a estudios de mujer, género y feminismo. Bounocore (p.88), ya en 1976 afirmaba lo siguiente "sabe más, no quien posee mayor cantidad de conocimientos, sino quien sabe dónde y cómo obtenerlos". Esta idea tiene total vigencia en la actualidad, en plena era de sobreabundancia de información, en donde sin las herramientas adecuadas, la persona interesada en un documento puede consumir mucho de su tiempo sin lograr dar con la información buscada.

Cabe mencionar que en noviembre de 2011 la UNC presentó, a través del Programa de Bibliotecas de la Universidad Nacional de Córdoba, el Repositorio Digital y el Portal de Revistas Científicas en el marco del Proyecto "Fortalecimiento de la Dimensión Internacional de la UNC", D/030237/10, realizado en cooperación con la Universidad Complutense de Madrid, el mismo es financiado por AECID.

\section{Bibliografía}

Buonocore, D. (1976). Diccionario de bibliotecología: Términos relativos a la bibliología, bibliografía, bibliofilia, biblioteconomía, archivología, documentología, tipografía y materias afines. Buenos Aires: Ediciones Marymar.

Huyer, S.; Westholm, G. (2002). Toolkit on gender indicators in engineering, science and technology. Paris: UNESCO. http://gstgateway. wigsat.org/toolkit/ch1.html

Torres Ramírez, I. de (2000). Los recursos informativo-documentales para los Estudios de las Mujeres. Panorámica breve desde Europa. http://www.nodo50.org/mujeresred/isabel de torres.htm\#3

Torres Ramírez, I. de (2003). "Los estudios de género y los recursos informativo-documentales que originan: síntomas evidentes del nuevo protagonismo de las mujeres. En: II Encuentro nacional de la red de Bibliotecas y Centros de Documentación especializados en Género y Mujeres. México, 2003. www.sabiduriaaplicada.com/documentos/los-estudios-de-genero.pdf

Torres Ramírez, I. de (2005). Tesis doctorales sobre estudios de las mujeres en España (1976-2002). A propósito de un indicador definitivo en investigación. En: Revista Española de Documentación Científica, 28(4), p.479-499

\section{Notas}

$1 \mathrm{http}: / /$ databases.unesco.org/thessp/

2 http://www.oei.es/genero/documentos/bibliografia/Bib_5.pdf

3 http://www.slais.ubc.ca/courses/libr517/02-03wt2/projects/berman/passions.htm 
Biblios Tratamiento de la investigación científica sobre los estudios de mujer ...

\section{Datos del autor}

María Eugenia Salvai

Licenciada en Bibliotecología y Documentación por la Universidad Nacional de Córdoba. A cargo de la biblioteca Ing. Jorge Domínguez - INTI Centro Regional Córdoba.

eugesalvai@gmail.com

Recibido-Received : 2013-03-03

Aceptado-Accepted : 2013-03-30

(cc) EY New articles in this journal are licensed under a Creative Commons Attribution 3.0 United States License.

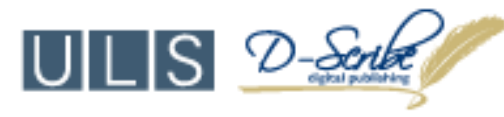

This journal is published by the University Library System of the University of Pittsburgh as part of its D-Scribe Digital Publishing Program and is cosponsored by the University of Pittsburgh Press. 